\title{
The clinical course of pain and disability following surgery for spinal stenosis: a systematic review and meta-analysis of cohort studies
}

\author{
Carolina G. Fritsch ${ }^{1,2}$ (D) Manuela L. Ferreira ${ }^{3,4}$ - Christopher G. Maher $^{3}$. \\ Robert D. Herbert ${ }^{5} \cdot$ Rafael Z. Pinto $^{6} \cdot$ Bart Koes $^{7} \cdot$ Paulo H. Ferreira $^{1}$
}

Received: 18 February 2016/Revised: 12 June 2016/Accepted: 16 June 2016/Published online: 21 July 2016

(c) Springer-Verlag Berlin Heidelberg 2016

\begin{abstract}
Purpose The aim of this study was to assess the clinical course of pain and disability in patients with lumbar spinal stenosis following surgery.

Methods Electronic databases were searched to July 2014 and only prospective cohort studies assessing pain or disability following surgery for lumbar spinal stenosis were included. Two independent reviewers extracted data and assessed study quality. Estimates of pain and disability (expressed as $0-100$ point scales) as well as $95 \%$
\end{abstract}

Systematic review registration: PROSPERO 2013: CRD42013005988.

Electronic supplementary material The online version of this article (doi:10.1007/s00586-016-4668-0) contains supplementary material, which is available to authorized users.

Carolina G. Fritsch

carolinafritsch@gmail.com

1 Discipline of Physiotherapy, Faculty Health Sciences, The University of Sydney, Sydney, Australia

2 Departamento de Fisioterapia, Universidade Federal de Ciências da Saúde de Porto Alegre, R. Sarmento Leite 245, Porto Alegre, Brazil

3 The George Institute for Global Health, Sydney Medical School, University of Sydney, Sydney, Australia

4 The Institute of Bone and Joint Research, Sydney Medical School, University of Sydney, Sydney, Australia

5 Neuroscience Research Australia, University of New South Wales, Sydney, Australia

6 Departamento de Fisioterapia, Faculdade de Ciências e Tecnologia, UNESP, Univ Estadual Paulista, Presidente Prudente, Brazil

7 Department of General Practice, Erasmus MC, Rotterdam, The Netherlands confidence intervals were obtained using meta-regression. The effect of time was clearly non-linear, so it was modelled using fractional polynomial regression.

Results From a total of 10,741 titles, 69 publications (64 cohort studies) were included in the review. Pooled estimate for pain pre-operatively was 63.4 (95\% CI 56.5; 70.3 ), reducing to 33.1 (95\% CI $24.2 ; 41.9)$ at 3 months and 19.2 points $(95 \%$ CI $9.2 ; 29.3)$ at 60 months. Preoperative estimates of disability were 36.9 (95\% CI 32.6; 41.3), reducing to $16.3(95 \% \mathrm{CI} 11.8 ; 20.9)$ at 3 months and $12.4(95 \%$ CI $7.7 ; 17.2)$ at 60 months.

Conclusion Patients with lumbar spinal stenosis experience rapid symptom reduction after surgery, but should still expect to experience mild-to-moderate pain and disability 60 months later.

Keywords Lumbar spinal stenosis - Meta-analysis · Prognosis $\cdot$ Surgery

\section{Background}

Lumbar spinal stenosis is a well recognised and severely debilitating spinal condition. It is generally attributed to a narrowing of the spinal canal, nerve root canals, or intervertebral foramina, usually as a consequence of age-related degenerative changes to the spine anatomy, including bone, ligaments, facet joints, and intervertebral disc. Clinical symptoms are believed to result from compression and/or ischaemia of vascular and neurological tissues in the spine. Neurogenic claudication is the most typical symptom, and may be accompanied by lower limb pain, numbness, paraesthesia, or weakness, usually exacerbated by standing or walking $[1,2]$. The available literature on the subject highlights that symptoms are exacerbated by extended 
positions or walking and relieved by flexed positions or sitting on patients with radiologically confirmed lumbar spinal stenosis [2]. Patients with lumbar spinal stenosis have greater mobility limitation than patients with knee or hip osteoarthritis, and this results in important reductions in both functional ability and quality-of-life [3].

Lumbar spinal stenosis has become a commonly diagnosed and treated condition of the spine-it is estimated that approximately one-fifth of adults aged 65 years or older will have symptoms of neurogenic claudication due to severe lumbar spinal stenosis [4, 5]; this condition becoming the most common reason for individuals older than 65 years undergoing spinal surgery $[1,6]$. In fact, surgical management has become the standard procedure in the management of symptomatic lumbar spinal stenosis, and as a result, this procedure is currently the fastest growing surgical procedure worldwide [6, 7]. Recent report yielded various degrees of clinical symptoms in relation to radiological findings [5], which highlights the importance of correlation of both clinical and radiological findings when targeting the surgical procedures. A recent systematic review of randomised clinical trials of surgery for lumbar spinal stenosis has shown that although patients will experience decreases in pain and disability following surgery, over a quarter will have further spinal surgery 1 year after having spinal surgery, suggesting they may not experience full recovery [8]. Moreover, recent studies have shown that there is no correlation between the severity of clinical symptoms and dural cross-sectional areas $[9,10]$ or between increased canal diameter following surgery and symptom improvement (i.e., back or leg pain, functional status, and neurological claudication) [11]. It is, therefore, also unclear whether the initial benefits of surgery in terms of pain and disability are sustained over the years, or whether patients will experience increase in symptoms over time. However, randomised clinical trials are not the ideal design to infer long-term course, as they usually provide shorter follow-up data and more stringent inclusion criteria, if compared to cohort studies.

$\mathrm{We}$, therefore, aimed to systematically review the literature to identify cohort studies assessing the long-term course of pain and disability in patients with lumbar spinal stenosis who have undergone surgery. To our knowledge, this is the first systematic review to assess the clinical course of lumbar spinal stenosis managed surgically.

\section{Methods}

\section{Data sources and searches}

This review was prospectively registered on PROSPERO (registration number CRD42013005988). MEDLINE,
CINAHL, and Embase databases were searched from inception to July 2014, to identify eligible studies. Search terms are available in Additional File 1. In addition to the electronic searches, citation tracking was conducted and the reference lists of the included studies in relevant systematic reviews were checked.

\section{Study selection}

No language or geographic restrictions were included in the search strategy, but non-English studies were included in the review only when translation was available. Independent reviewers screened titles and abstracts (CF, MF, CGM, and PHF) for inclusion. The full text of potentially eligible studies was then obtained and assessed by three independent reviewers $(\mathrm{CF}, \mathrm{MF}$, and $\mathrm{RP})$ for inclusion against our criteria. Disagreements were resolved by consensus.

To be eligible for inclusion in the review studies needed to explicitly report that participants had a primary diagnosis of lumbar spinal stenosis of any duration. Diagnosis had to be defined either by imaging techniques or clinically by the presence of signs and symptoms.

All prospective surgical cohort studies with at least 3-month follow-up that reported pain or disability outcomes were included. Studies that only reported recovery rates or percentage change in pain or disability were excluded.

\section{Data extraction}

For each study, summary data were obtained on sample source, sample size, patient characteristics, outcomes (pain and disability), duration of follow-up, and inception time, if applicable. Measures of central tendency (e.g., mean or median) and dispersion (e.g., standard deviation, standard error or $95 \%$ confidence intervals) were extracted for pain and disability outcomes. Outcome data were re-scaled to a common 0-100 scale to facilitate between-study comparisons (e.g., means and standard deviations of pain scores given on a $0-10$-point scale were multiplied by 10 ; means and standard deviations of disability scores given on a 24 -point scale were multiplied by 4.1666 or $100 / 24)$. When insufficient data were reported on outcome measures, authors were contacted by e-mail for further details. If data on dispersion were not provided by the authors, standard deviations were imputed from similar studies.

\section{Quality assessment}

Methodological quality of included studies was assessed using an adaptation of the methodological criteria suggested by Altman [12] and include two items on sampling, two on completeness of follow-up, and one item on 
description of prognostic outcomes. These criteria have been used in a previous systematic review on prognosis of low back pain [13]. The results of the methodological quality assessment for each study are presented as percentage (Table 1).

\section{Data synthesis}

To accommodate the different time points for outcome assessment in the included studies, pain and disability were modelled as a continuous function of time. For all analyses, time was treated as time from surgery. If studies reported more than one measure of pain intensity (e.g., back and leg pain), the more severe measure at baseline was included in the analyses. In addition, secondary analyses were performed for back pain and leg pain separately.

Pooled estimates of outcomes were obtained using generalised estimating equations to account for the dependence of repeated observations (follow-ups) within studies. The observations from each study were assigned a weight equal to the inverse square of the mean SE of all observations from that study. The effect of time was clearly non-linear, so it was modelled using fractional polynomial regression [14]. The regression models were used to generate pooled point and interval estimates of outcomes at baseline and 3, 6, 12, 24, and 60 months.

\section{Results}

From a total of 10,741 titles, 69 publications reporting on 64 cohort studies were included in the review (references [15-17] are multiple publications reporting follow-up assessments of the same cohort, as are [18, 19] and [20, 21]). These studies provided data on 3774 participants (Fig. 1). Table 1 presents the main characteristics of all included studies (complete references of included studies can be found in Additional File 2). In more than $50 \%$ of the included studies, participants had persistent symptoms of lumbar spinal stenosis $(n=38 ; 57 \%)$. For the remaining 31 studies, symptom duration was not reported. In approximately one-fifth of the included studies, the recruited participants had central canal stenosis $(n=12$ studies), whereas most studies $(n=55$ studies; $80 \%)$ included a mixed population (i.e., central and lateral stenosis); and $3 \%$ (two studies) included only participants with a diagnosis of lateral canal stenosis. In most studies, the main complaint of included participants was intermittent neurological claudication with or without pain $(n=42$ studies; $61 \%$ ), followed by low back pain with or without leg pain $(n=11$ studies; $16 \%)$ and radicular pain only $(n=3$ studies; $4 \%)$. In 13 studies (19\%), main complaints were not reported or unclear.

\section{Methodological quality}

All included studies presented follow-up data for at least one outcome measure at 3 months or later $(n=69)$, but only approximately half $(n=40 ; 58 \%)$ reported enough data on clinical prognosis of lumbar spine stenosis to be included in the meta-analyses. In over three quarters of the studies, the follow-up included at least $80 \%$ of the sample ( $n=55$ studies or $81 \%$ ), however, only one-third of included studies ( $n=25$ studies or $37 \%$ ) clearly included a representative sample of participants with spinal stenosis and in only $61 \%$ of the studies was the sample well defined (i.e., inclusion and exclusion criteria provided).

\section{Clinical course of pain and disability}

Of the 64 included studies (69 publications), 31 provided sufficient data on disability and 39 provided sufficient data on pain to be included in the meta-analysis. Follow-up time ranged from 3 to 72 months post-surgery. In all except one study [22], baseline assessments were performed pre-operatively at the time of hospital admission. Surgical admission was, therefore, regarded as the inception time in the mixed-model analyses. The study by Bednar [22] which did not report pain or disability at the time of surgical admission was excluded from the analyses. Ha et al. [23] did not report enough data to be included in the analyses and was also excluded.

Pain and disability outcomes are presented in Fig. 2a and $b$, respectively. At inception (i.e., pre-operatively), the mean weighted pain score across all cohort studies was 63.4 (95\% CI 56.5-70.3). At 3-month post-surgery, pain had decreased to a weighted mean of 33.1 (95\% CI 24.2-41.9). Little further reduction in pain was seen at 6 months (mean 28.2; $95 \%$ CI 19.1-37.4), 12 months (mean 24.5; $95 \%$ CI 15.0-34.0), 24 months (mean 21.8; $95 \%$ CI 12.0-31.5), or 60 months (mean 19.2; $95 \%$ CI 9.2-29.3). The mean weighted disability scores at baseline were 36.9 (95\% CI 32.6-41.3), decreasing to 16.3 (95\% CI 11.8-20.9) at 3 months, 14 (95\% CI 9.3-18.6) at 6 months, 12.9 (95\% CI 8.2-17.6) at 12 months, 12.6 (95\% CI 7.8-17.3) at 24 months, and $12.4(95 \% \mathrm{CI}$ 7.7-17.2) at 60 months.

The mean standard deviation at baseline was 16.5 (range 6.0-30.3) for pain and 17.3 (range 4.1-62.0) for disability. This indicates a moderate degree of person-to-person variability in outcomes within studies.

At inception, the mean weighted leg pain score was 53 points (95\% CI 43.9-62.2), decreasing to 17.6 (95\% CI 4.6-30.6) 3 months after surgery, and further decreasing at 6 (mean 17.0; $95 \%$ CI 4.0-30.1), 12 (mean 15.9; $95 \%$ CI 2.0-29.8), 24 (mean 13.6; $95 \% \mathrm{CI}-4.0$ to 31.3), and 60 months (mean 6.8 ; $95 \% \mathrm{CI}-28.7$ to 42.4 ). For back 
Table 1 Characteristics of included studies

\begin{tabular}{|c|c|c|c|c|c|c|c|c|}
\hline References & Country & $\begin{array}{l}\text { Diagnosis } \\
\text { of lumbar } \\
\text { spinal } \\
\text { stenosis }\end{array}$ & $\begin{array}{l}\text { Age of } \\
\text { participants } \\
\text { (years) }\end{array}$ & $\begin{array}{l}\text { No. of } \\
\text { participants } \\
\text { (study } \\
\text { entry) }\end{array}$ & $\begin{array}{l}\text { Symptom } \\
\text { duration at } \\
\text { study entry }\end{array}$ & Outcome measures & Follow-up & $\begin{array}{l}\text { Quality } \\
\text { analysis } \\
\text { score } \\
(\%)\end{array}$ \\
\hline $\begin{array}{l}\text { Aleem et al. } \\
\text { [33] }\end{array}$ & Canada & Mixed & $\begin{array}{l}<70: \\
\quad n=68 \\
>70: \\
n=41\end{array}$ & 109 & Not reported & Disability (ODI 0-100) & $\begin{array}{l}6 \text { weeks, } 6 \\
\text { and } \\
12 \text { months }\end{array}$ & 60 \\
\hline $\begin{array}{l}\text { Anjarwalla } \\
\text { et al. [34] }\end{array}$ & UK & Mixed & $\begin{array}{l}\text { Mean } 53 \\
\quad(\text { SD 14) }\end{array}$ & 72 & Not reported & $\begin{array}{l}\text { Pain (VAS 0-100), } \\
\text { disability (ODI 0-100) }\end{array}$ & $\begin{array}{l}6 \text { and } \\
12 \text { months }\end{array}$ & 60 \\
\hline $\begin{array}{l}\text { Athiviraham } \\
\text { et al. [35] }\end{array}$ & Canada & Mixed & Mean 66 & 88 & $\begin{array}{l}35 \% \\
\quad<12 \text { months }\end{array}$ & Disability (RMQ 0-14) & 24 months & 80 \\
\hline $\begin{array}{l}\text { Atlas et al. } \\
\text { [15] }\end{array}$ & USA & Mixed & $\begin{array}{l}\text { Mean } 65.7 \\
\quad(\text { SD 10.7) }\end{array}$ & 81 & $\begin{array}{l}61 \% \\
>6 \text { months }\end{array}$ & $\begin{array}{l}\text { Back and leg pain } \\
\text { (bothersomeness scale } \\
\text { 0-6), disability } \\
\text { (Modified RMQ 0-23) }\end{array}$ & $\begin{array}{l}3,6,9 \text {, and } \\
12 \text { months }\end{array}$ & 80 \\
\hline $\begin{array}{l}\text { Atlas et al. } \\
\text { [16] }\end{array}$ & USA & Mixed & $\begin{array}{l}\text { Mean } 65.7 \\
\quad(\text { SD 10.7) }\end{array}$ & 81 & $\begin{array}{l}61 \% \\
\quad>6 \text { months }\end{array}$ & $\begin{array}{l}\text { Back and leg pain } \\
\text { (bothersomeness scale } \\
\text { 0-6), disability } \\
\text { (Modified RMQ 0-23) }\end{array}$ & $\begin{array}{l}24,36 \text {, and } \\
48 \text { months }\end{array}$ & 80 \\
\hline $\begin{array}{l}\text { Atlas et al. } \\
\text { [17] }\end{array}$ & USA & Mixed & $\begin{array}{l}\text { Mean } 65 \\
\quad(\text { SD 10.7) }\end{array}$ & 81 & $\begin{array}{l}61 \% \\
>6 \text { months }\end{array}$ & $\begin{array}{l}\text { Back and leg pain } \\
\text { (bothersomeness scale } \\
\text { 0-6), disability } \\
\text { (Modified RMQ 0-23) }\end{array}$ & $\begin{array}{l}60,72,84,96, \\
108 \text { and } \\
120 \text { months }\end{array}$ & 80 \\
\hline Bednar [22] & Canada & Mixed & $\begin{array}{l}\text { Mean } 67 \\
\text { (range } \\
52-85)\end{array}$ & 56 & Not reported & $\begin{array}{l}\text { Pain (VAS 0-10), } \\
\text { disability (ODI 0-100) }\end{array}$ & $\begin{array}{l}24 \text { and } \\
33 \text { months }\end{array}$ & 60 \\
\hline $\begin{array}{l}\text { Beyer et al. } \\
{[36]}\end{array}$ & Germany & Mixed & $\begin{array}{l}\text { Mean } 69 \\
\quad(\text { SD 9.7) }\end{array}$ & 32 & Not reported & $\begin{array}{l}\text { Back and leg pain (VAS } \\
0-10 \text { ), disability (ODI } \\
0-100)\end{array}$ & $\begin{array}{l}12 \text { and } \\
24 \text { months }\end{array}$ & 60 \\
\hline $\begin{array}{l}\text { Bhadra et al. } \\
\text { [37] }\end{array}$ & UK & Mixed & $\begin{array}{l}\text { Mean 61 } \\
\text { (range } \\
52-94)\end{array}$ & 45 & $\begin{array}{l}70 \% \\
\quad<24 \text { months }\end{array}$ & $\begin{array}{l}\text { Back and leg pain (VAS } \\
0-10 \text { ), disability (ODI } \\
0-100)\end{array}$ & unclear & 80 \\
\hline $\begin{array}{l}\text { Castro- } \\
\text { Menendes } \\
\text { et al. [38] }\end{array}$ & Spain & Mixed & $\begin{array}{l}\text { Mean } 56 \\
\quad(\text { SD 10.2) }\end{array}$ & 50 & 30 months & $\begin{array}{l}\text { Back and leg pain (VAS } \\
0-10 \text { ), disability (ODI } \\
0-100)\end{array}$ & $\begin{array}{l}\text { 6, } 12 \text { and } \\
48 \text { months }\end{array}$ & 80 \\
\hline $\begin{array}{l}\text { Cavagna et al. } \\
\text { [39] }\end{array}$ & France & Mixed & $\begin{array}{l}\text { Mean } 73 \\
\quad \text { (range } \\
65-87 \text { ) }\end{array}$ & 39 & Not reported & $\begin{array}{l}\text { Back and leg pain (VAS } \\
0-100) \text {, disability } \\
\text { (ODI 0-100) }\end{array}$ & $\begin{array}{r}6,12,24 \text { and } \\
48 \text { months }\end{array}$ & 60 \\
\hline $\begin{array}{l}\text { Cavusoglu } \\
\text { et al. [40] }\end{array}$ & Turkey & Mixed & $\begin{array}{l}\text { Mean } 70 \\
\quad(\text { SD 15.1) }\end{array}$ & 50 & $\begin{array}{l}\text { Range } \\
\text { 9-58 months }\end{array}$ & $\begin{array}{l}\text { Pain (VAS 0-10), } \\
\text { disability (ODI 0-100) }\end{array}$ & $\begin{array}{l}3 \text { and } \\
22 \text { months }\end{array}$ & 60 \\
\hline $\begin{array}{l}\text { Chopko et al. } \\
\text { [41] }\end{array}$ & USA & Central & $\begin{array}{l}\text { Mean } 70 \\
\text { (range } \\
45-88)\end{array}$ & 45 & Not reported & $\begin{array}{l}\text { Pain (VAS 0-10), } \\
\quad \text { disability (ODI 0-100) }\end{array}$ & $\begin{array}{l}6,12 \text { and } \\
24 \text { months }\end{array}$ & 40 \\
\hline $\begin{array}{l}\text { Colak et al. } \\
\text { [42] }\end{array}$ & Turkey & Lateral & $\begin{array}{c}\text { Mean } 52 \\
\text { (range } \\
42-71)\end{array}$ & 16 & 53 months & Pain (VAS 0-10) & $\begin{array}{l}3 \text { and } \\
12 \text { months }\end{array}$ & 80 \\
\hline $\begin{array}{l}\text { Datta et al. } \\
\text { [43] }\end{array}$ & UK & Mixed & $\begin{array}{r}\text { Mean } 62 \\
\quad(\text { SD 4) }\end{array}$ & 20 & 35 months & $\begin{array}{l}\text { Back pain (VAS } 0-10), \\
\text { disability (ODI } 0-100)\end{array}$ & 6 months & 60 \\
\hline $\begin{array}{l}\text { Deer et al. } \\
\text { [44] }\end{array}$ & USA & Central & $\begin{array}{l}\text { Mean } 66 \\
\text { (range } \\
46-80)\end{array}$ & 35 & Not reported & $\begin{array}{l}\text { Pain (VAS 0-10), } \\
\text { disability (ODI 0-100) }\end{array}$ & $\begin{array}{l}3,6 \text { and } \\
12 \text { months }\end{array}$ & 60 \\
\hline $\begin{array}{l}\text { Delank et al. } \\
\text { [45] }\end{array}$ & Germany & Mixed & Mean 68 & 13 & Not reported & $\begin{array}{l}\text { Pain (VAS 0-10), } \\
\text { disability (ODI 0-100) }\end{array}$ & $\begin{array}{l}6 \text { and } \\
12 \text { months }\end{array}$ & 80 \\
\hline $\begin{array}{l}\text { El-Abed et al. } \\
\text { [46] }\end{array}$ & UK & Mixed & $\begin{array}{l}\text { Mean } 64 \\
\quad(\text { SD 16.2) }\end{array}$ & 120 & $\geq 3$ months & $\begin{array}{l}\text { Pain (VAS 0-10), } \\
\text { disability (ODI 0-100) }\end{array}$ & $\begin{array}{l}6 \text { and } \\
36 \text { months }\end{array}$ & 80 \\
\hline
\end{tabular}


Table 1 continued

\begin{tabular}{|c|c|c|c|c|c|c|c|c|}
\hline References & Country & $\begin{array}{l}\text { Diagnosis } \\
\text { of lumbar } \\
\text { spinal } \\
\text { stenosis }\end{array}$ & $\begin{array}{l}\text { Age of } \\
\text { participants } \\
\text { (years) }\end{array}$ & $\begin{array}{l}\text { No. of } \\
\text { participants } \\
\text { (study } \\
\text { entry) }\end{array}$ & $\begin{array}{l}\text { Symptom } \\
\text { duration at } \\
\text { study entry }\end{array}$ & Outcome measures & Follow-up & $\begin{array}{l}\text { Quality } \\
\text { analysis } \\
\text { score } \\
(\%)\end{array}$ \\
\hline Endres [47] & Germany & Mixed & $\begin{array}{l}\text { Mean } 80 \\
\text { (range } \\
73-88)\end{array}$ & 58 & Not reported & $\begin{array}{l}\text { Pain (VAS } 0-10) \\
\quad \text { disability (ODI } 0-100)\end{array}$ & 46 months & 60 \\
\hline $\begin{array}{l}\text { Frazier et al. } \\
\text { [48] }\end{array}$ & USA & Mixed & $\begin{array}{l}\text { Mean } 67 \\
\quad \text { range } \\
52-90)\end{array}$ & 90 & Not reported & $\begin{array}{l}\text { Back and leg pain (VAS } \\
0-10)\end{array}$ & 6 months & 60 \\
\hline Fu et al. [49] & China & Mixed & $\begin{array}{l}\text { Mean } 57 \\
\text { (range } \\
45-73)\end{array}$ & 152 & Not reported & $\begin{array}{l}\text { Back and leg pain (VAS } \\
0-10 \text { ), disability (ODI } \\
0-100)\end{array}$ & 40 months & 100 \\
\hline $\begin{array}{l}\text { Greiner-Perth } \\
\text { et al. [50] }\end{array}$ & Germany & $\begin{array}{l}\text { Central, } \\
\text { lateral } \\
\text { and } \\
\text { mixed }\end{array}$ & Mean 73 & 17 & Not reported & $\begin{array}{l}\text { Back and leg pain (VAS } \\
0-10 \text { ) }\end{array}$ & 34 months & 80 \\
\hline Ha et al. [23] & Korea & Mixed & $\begin{array}{l}\text { Mean } 63 \\
\quad(\text { SD 9.0) }\end{array}$ & 31 & Not reported & $\begin{array}{l}\text { Back and leg pain (VAS } \\
0-10 \text { ), disability (ODI } \\
0-100)\end{array}$ & $\begin{array}{r}3,6,12,24 \\
31 \text { months }\end{array}$ & 80 \\
\hline $\begin{array}{l}\text { Haro et al. } \\
\text { [51] }\end{array}$ & Japan & Central & $\begin{array}{l}\text { Mean } 67 \\
\quad(\text { SD 10.9) }\end{array}$ & 42 & $\geq 6$ months & $\begin{array}{l}\text { Back and leg pain (VAS } \\
0-100) \text {, disability } \\
\text { (ODI 0-100) }\end{array}$ & 24 months & 80 \\
\hline $\begin{array}{c}\text { Herno et al. } \\
{[52]}\end{array}$ & Finland & Mixed & $\begin{array}{l}\text { Mean 51 } \\
\text { (range } \\
22-67)\end{array}$ & 108 & 115 months & Disability (ODI 0-100) & $\begin{array}{l}82 \text {, } \\
154 \text { months }\end{array}$ & 60 \\
\hline $\begin{array}{l}\text { Ikuta et al. } \\
\text { [53] }\end{array}$ & Japan & Mixed & Mean 69 & 37 & 40 months & Disability (RM 0-24) & 38 months & 60 \\
\hline $\begin{array}{l}\text { Jakola et al. } \\
{[54]}\end{array}$ & Norway & Mixed & $\begin{array}{l}\text { Mean } 75 \\
\quad(\text { SD } 4.1)\end{array}$ & 101 & 100 weeks & $\begin{array}{l}\text { Back and leg pain (VAS } \\
0-100) \text {, disability } \\
\text { (ODI 0-100) }\end{array}$ & $\begin{array}{l}3 \text { and } \\
12 \text { months }\end{array}$ & 60 \\
\hline $\begin{array}{l}\text { Kaner et al. } \\
{[55]}\end{array}$ & Turkey & Mixed & $\begin{array}{l}\text { Mean } 67 \\
\text { (range } \\
40-85 \text { ) }\end{array}$ & 30 & $\geq 12$ months & $\begin{array}{l}\text { Pain (VAS 0-10), } \\
\quad \text { disability (ODI 0-100) }\end{array}$ & $\begin{array}{l}3,12 \text { and } \\
24 \text { months }\end{array}$ & 80 \\
\hline $\begin{array}{c}\text { Kim et al. } \\
{[56]}\end{array}$ & Korea & Mixed & Mean 70 & 23 & Not reported & $\begin{array}{l}\text { Pain (VAS 0-10), } \\
\text { disability (ODI 0-100) }\end{array}$ & 17.5 months & 60 \\
\hline$\underset{\text { Kim et al. }}{\text { K57] }}$ & Korea & Mixed & $\begin{array}{l}\text { Mean } 78 \\
\text { (range } \\
75-82 \text { ) }\end{array}$ & 14 & $\geq 3$ months & $\begin{array}{l}\text { Pain (VAS 0-10), } \\
\text { disability (ODI 0-100) }\end{array}$ & 17.5 months & 80 \\
\hline $\begin{array}{l}\text { Komp et al. } \\
{[58]}\end{array}$ & Germany & Central & $\begin{array}{l}\text { Mean 61 } \\
\text { (range } \\
43-81)\end{array}$ & 90 & 15 months & $\begin{array}{l}\text { Back and leg pain (VAS } \\
0-100) \text {, disability } \\
\text { (ODI 0-100) }\end{array}$ & $\begin{array}{r}3,6,12 \text { and } \\
24 \text { months }\end{array}$ & 60 \\
\hline $\begin{array}{l}\text { Kong et al. } \\
\text { [59] }\end{array}$ & Korea & Mixed & $\begin{array}{l}\text { Mean 58 } \\
\text { (range } \\
38-78)\end{array}$ & 42 & Not reported & $\begin{array}{l}\text { Back and leg pain (VAS } \\
0-10 \text { ), disability (ODI } \\
0-100)\end{array}$ & 12 months & 80 \\
\hline $\begin{array}{l}\text { Kuchta et al. } \\
\text { [60] }\end{array}$ & Germany & Mixed & $\begin{array}{c}\text { Mean } 69 \\
\text { (range } \\
41-91)\end{array}$ & 175 & Not reported & $\begin{array}{l}\text { Leg pain (VAS 0-100), } \\
\text { disability (ODI 0-100) }\end{array}$ & $\begin{array}{l}\text { 6, } 12 \text { and } \\
24 \text { months }\end{array}$ & 60 \\
\hline $\begin{array}{l}\text { Mannion } \\
\text { et al. [61] }\end{array}$ & $\begin{array}{l}\text { Australia/ } \\
\text { UK }\end{array}$ & Central & $\begin{array}{l}\text { Mean } 70 \\
\text { (range } \\
43-88)\end{array}$ & 50 & Not reported & Disability (ODI 0-100) & $\begin{array}{r}3,6,12 \text { and } \\
24 \text { months }\end{array}$ & 40 \\
\hline $\begin{array}{l}\text { Mekhail et al. } \\
\text { (62) }\end{array}$ & USA & Central & $\begin{array}{l}\text { Mean } 72 \\
\text { (range } \\
53-86)\end{array}$ & 34 & 60 months & $\begin{array}{l}\text { Pain (VAS 0-10), } \\
\text { disability (RMQ 0-24) }\end{array}$ & $\begin{array}{r}6,9,12 \text { and } \\
24 \text { months }\end{array}$ & 80 \\
\hline $\begin{array}{l}\text { Mekhail et al. } \\
\text { (63) }\end{array}$ & USA & Mixed & $\begin{array}{l}\text { Mean } 70 \\
\text { (range } \\
45-88)\end{array}$ & 58 & $\begin{array}{l}76 \% \\
>6 \text { months }\end{array}$ & $\begin{array}{l}\text { Pain (VAS 0-10), } \\
\text { disability (ODI 0-100) }\end{array}$ & 12 months & 80 \\
\hline
\end{tabular}


Table 1 continued

\begin{tabular}{|c|c|c|c|c|c|c|c|c|}
\hline References & Country & $\begin{array}{l}\text { Diagnosis } \\
\text { of lumbar } \\
\text { spinal } \\
\text { stenosis }\end{array}$ & $\begin{array}{l}\text { Age of } \\
\text { participants } \\
\text { (years) }\end{array}$ & $\begin{array}{l}\text { No. of } \\
\text { participants } \\
\text { (study } \\
\text { entry) }\end{array}$ & $\begin{array}{l}\text { Symptom } \\
\text { duration at } \\
\text { study entry }\end{array}$ & Outcome measures & Follow-up & $\begin{array}{l}\text { Quality } \\
\text { analysis } \\
\text { score } \\
(\%)\end{array}$ \\
\hline $\begin{array}{l}\text { Mlyavykh } \\
\text { et al. [93] }\end{array}$ & Russia & Mixed & $\begin{array}{l}\text { Mean } 61 \\
\text { (range } \\
47-71)\end{array}$ & 19 & $\geq 6$ months & $\begin{array}{l}\text { Back and leg pain (VAS } \\
0-10 \text { ), disability (ODI } \\
0-100)\end{array}$ & 12 months & 80 \\
\hline $\mathrm{Ng}$ et al. [64] & UK & Central & $\begin{array}{l}\text { Mean } 62 \\
\text { (range } \\
55-82)\end{array}$ & 100 & 73 months & $\begin{array}{l}\text { Pain (VAS 0-10), } \\
\quad \text { disability (ODI } 0-100)\end{array}$ & $\begin{array}{l}12 \text { and } \\
24 \text { months }\end{array}$ & 100 \\
\hline $\begin{array}{l}\text { Ohtori et al. } \\
{[65]}\end{array}$ & Japan & Central & $\begin{array}{l}\text { Mean } 65 \\
\text { (range } \\
55-80)\end{array}$ & 33 & 36 months & $\begin{array}{l}\text { Back pain (VAS 0-10), } \\
\text { disability (ODI } 0-100)\end{array}$ & 24 months & 80 \\
\hline $\begin{array}{l}\text { Paker et al. } \\
\text { [66] }\end{array}$ & Turkey & Mixed & $\begin{array}{l}\text { Mean } 65 \\
\quad(\text { SD } 7.3)\end{array}$ & 22 & Not reported & Pain (VAS 0-10) & 18 months & 60 \\
\hline $\begin{array}{l}\text { Palmer et al. } \\
\text { [67] }\end{array}$ & USA & Mixed & $\begin{array}{c}\text { Mean } 67 \\
\text { (range } \\
43-84)\end{array}$ & 54 & Not reported & $\begin{array}{l}\text { Back and leg pain (VAS } \\
0-10)\end{array}$ & $\begin{array}{l}3,6.5 \text { and } \\
11.5 \text { months }\end{array}$ & 60 \\
\hline $\begin{array}{l}\text { Panagiotis } \\
\text { et al. [68] }\end{array}$ & Greece & Mixed & $\begin{array}{l}\text { Mean 61 } \\
\text { (range } \\
33-79)\end{array}$ & 41 & $\geq 6$ months & $\begin{array}{l}\text { Pain (VAS 0-10), } \\
\quad \text { disability (ODI } 0-100)\end{array}$ & $\begin{array}{l}12,24,36,48, \\
60 \text { and } \\
72 \text { months }\end{array}$ & 40 \\
\hline Pao et al. [69] & Taiwan & Mixed & $\begin{array}{c}\text { Mean 62 } \\
\quad \text { range } \\
36-86)\end{array}$ & 60 & $\geq 3$ months & Disability (ODI 0-100) & 15.7 months & 100 \\
\hline $\begin{array}{l}\text { Papavero } \\
\text { et al. [70] }\end{array}$ & Germany & Mixed & $\begin{array}{l}\text { Median age } \\
\text { (range } \\
58.1-80.3 \text { ) }\end{array}$ & 165 & $\geq 3$ months & Pain (VAS 0-10) & $\begin{array}{l}3 \text { and } \\
12 \text { months }\end{array}$ & 80 \\
\hline $\begin{array}{l}\text { Parker et al. } \\
\text { [71] }\end{array}$ & USA & Mixed & $\begin{array}{l}\text { Mean } 57 \\
\quad(\text { SD 11) }\end{array}$ & 54 & $\geq 6$ months & $\begin{array}{l}\text { Leg pain (VAS } 0-10) \text {, } \\
\text { disability (ODI } 0-100)\end{array}$ & 24 months & 100 \\
\hline $\begin{array}{l}\text { Parlato et al. } \\
\text { [72] }\end{array}$ & Italy & Mixed & $\begin{array}{r}\text { Mean } 49.6 \\
\quad(\text { SD 13.4) }\end{array}$ & 58 & Not reported & Pain (VAS 0-10) & $\begin{array}{l}3 \text { and } \\
12 \text { months }\end{array}$ & 100 \\
\hline $\begin{array}{l}\text { Postacchini } \\
\text { et al. [73] }\end{array}$ & Italy & Mixed & $\begin{array}{l}\text { Mean } 65 \\
\text { (range } \\
53-81)\end{array}$ & 66 & 8 months & Disability (ODI 0-100) & $\begin{array}{l}3,6 \text { and } \\
24 \text { months }\end{array}$ & 80 \\
\hline $\begin{array}{l}\text { Reyes- } \\
\text { Sanchez } \\
\text { et al. [74] }\end{array}$ & Mexico & Mixed & $\begin{array}{l}\text { Mean } 44 \\
\text { (range } \\
24-60)\end{array}$ & 20 & Not reported & $\begin{array}{l}\text { Back and leg pain (VAS } \\
0-10 \text { ), disability (ODI } \\
0-100)\end{array}$ & 24 months & 100 \\
\hline $\begin{array}{l}\text { Richter et al. } \\
\text { [19] }\end{array}$ & Germany & Mixed & $\begin{array}{l}\text { Mean } 68.3 \\
\text { (range } \\
49-79)\end{array}$ & 60 & $\geq 3$ months & $\begin{array}{l}\text { Pain (VAS 0-10), } \\
\text { disability (ODI 0-100; } \\
\text { RMQ 0-24) }\end{array}$ & $\begin{array}{l}3,6 \text { and } \\
12 \text { months }\end{array}$ & 80 \\
\hline $\begin{array}{l}\text { Richter et al. } \\
\text { [18] }\end{array}$ & Germany & Mixed & $\begin{array}{l}\text { Mean } 68 \\
\text { (range } \\
52-79)\end{array}$ & 31 & $\geq 3$ months & $\begin{array}{l}\text { Pain (VAS 0-10), } \\
\text { disability (ODI 0-100; } \\
\text { RMQ 0-24) }\end{array}$ & 24 months & 60 \\
\hline $\begin{array}{l}\text { Schaeren } \\
\text { et al. [20] }\end{array}$ & Switzerland & Mixed & $\begin{array}{l}\text { Mean } 71 \\
\text { (range } \\
47-87 \text { ) }\end{array}$ & 26 & 35 months & Pain (VAS 0-100) & 52 months & 60 \\
\hline $\begin{array}{l}\text { Schnake et al. } \\
\text { [21] }\end{array}$ & Switzerland & Mixed & $\begin{array}{l}\text { Mean } 71 \\
\quad \text { range } \\
47-87)\end{array}$ & 26 & 35 months & Pain (VAS 0-100) & 24 months & 80 \\
\hline $\begin{array}{l}\text { Schulte et al. } \\
\text { [75] }\end{array}$ & Germany & Central & $\begin{array}{l}\text { Mean } 69 \\
\quad(\text { SD } 7.5)\end{array}$ & 50 & $\geq 3$ months & $\begin{array}{c}\text { Back and leg pain (VAS } \\
0-10 \text { ), disability (ODI } \\
0-100 \text {; RMQ 0-24) }\end{array}$ & $\begin{array}{l}3 \text { and } \\
12 \text { months }\end{array}$ & 100 \\
\hline $\begin{array}{l}\text { Shabat et al. } \\
\text { [76] }\end{array}$ & Israel & Mixed & $\begin{array}{l}\text { Mean } 84 \\
\text { (range } \\
80-91)\end{array}$ & 25 & 53 months & Pain (VAS 0-10) & 37 months & 40 \\
\hline $\begin{array}{l}\text { Shabat et al. } \\
\text { [77] }\end{array}$ & US & Mixed & $\begin{array}{l}\text { Mean } 70 \\
\quad(\text { SD 11) }\end{array}$ & 53 & 30 months & $\begin{array}{l}\text { Back and leg pain (VAS } \\
0-10 \text { ), disability (ODI } \\
0-100)\end{array}$ & $\begin{array}{l}12 \text { and } \\
24 \text { months }\end{array}$ & 60 \\
\hline
\end{tabular}


Table 1 continued

\begin{tabular}{|c|c|c|c|c|c|c|c|c|}
\hline References & Country & $\begin{array}{l}\text { Diagnosis } \\
\text { of lumbar } \\
\text { spinal } \\
\text { stenosis }\end{array}$ & $\begin{array}{l}\text { Age of } \\
\text { participants } \\
\text { (years) }\end{array}$ & $\begin{array}{l}\text { No. of } \\
\text { participants } \\
\text { (study } \\
\text { entry) }\end{array}$ & $\begin{array}{l}\text { Symptom } \\
\text { duration at } \\
\text { study entry }\end{array}$ & Outcome measures & Follow-up & $\begin{array}{l}\text { Quality } \\
\text { analysis } \\
\text { score } \\
(\%)\end{array}$ \\
\hline $\begin{array}{l}\text { Sigmundsson } \\
\text { et al. [78] }\end{array}$ & Sweden & Central & $\begin{array}{l}\text { Mean } 71 \\
\quad(\text { SD 10) }\end{array}$ & 109 & Not reported & $\begin{array}{l}\text { Back and leg pain (VAS } \\
0-100) \text {, disability } \\
\text { (ODI 0-100) }\end{array}$ & 12 months & 100 \\
\hline $\begin{array}{l}\text { Sinikallio } \\
\text { [79] }\end{array}$ & Finland & Mixed & $\begin{array}{l}\text { Mean } 62 \\
\quad(\mathrm{SD} \\
11.84)\end{array}$ & 96 & Not reported & $\begin{array}{l}\text { Pain (VAS 0-100), } \\
\text { disability (ODI 0-100) }\end{array}$ & $\begin{array}{r}3,6,12 \text { and } \\
24 \text { months }\end{array}$ & 80 \\
\hline $\begin{array}{l}\text { Sobottke } \\
\text { et al. [80] }\end{array}$ & Germany & Mixed & $\begin{array}{l}\text { Mean } 68 \\
\quad(\text { SD } 9.7)\end{array}$ & 29 & Not reported & $\begin{array}{l}\text { Back and leg pain (VAS } \\
0-10 \text { ), disability (ODI } \\
0-100)\end{array}$ & $\begin{array}{l}6 \text { and } \\
12 \text { months }\end{array}$ & 80 \\
\hline $\begin{array}{l}\text { Stromqvist } \\
\text { et al. [81] }\end{array}$ & Sweden & $\begin{array}{l}\text { Central } \\
\text { and } \\
\text { lateral }\end{array}$ & $\begin{array}{c}\text { Mean } 69 \\
\text { (range } \\
49-89)\end{array}$ & 140 & $\begin{array}{l}41 \% \\
\quad>24 \text { months }\end{array}$ & $\begin{array}{l}\text { Back and leg pain (VAS } \\
0-100)\end{array}$ & $\begin{array}{l}12,24 \text { and } \\
60 \text { months }\end{array}$ & 40 \\
\hline $\begin{array}{l}\text { Surace et al. } \\
\text { [82] }\end{array}$ & Italy & Lateral & $\begin{array}{l}\text { Mean 64 } \\
\text { (range } \\
45-88)\end{array}$ & 35 & Not reported & Pain (VAS 0-10) & 23 months & 40 \\
\hline $\begin{array}{l}\text { Tenhula et al. } \\
\text { [83] }\end{array}$ & US & Mixed & $\begin{array}{l}\text { Mean } 68 \\
\quad(\text { SD } 8.7)\end{array}$ & 32 & Not reported & $\begin{array}{l}\text { Pain (VAS 0-10), } \\
\quad \text { disability (ODI 0-100) }\end{array}$ & $\begin{array}{l}\text { 6, } 12 \text { and } \\
24 \text { months }\end{array}$ & 60 \\
\hline $\begin{array}{l}\text { Westergaard } \\
\text { et al. [84] }\end{array}$ & Denmark & Mixed & $\begin{array}{r}\text { Median } 70 \\
\text { (IQR 19) }\end{array}$ & 146 & $\begin{array}{l}80 \% \\
\quad \geq 12 \text { months }\end{array}$ & Disability (ODI 0-100) & $\begin{array}{r}3,6,12 \text { and } \\
24 \text { months }\end{array}$ & 60 \\
\hline $\begin{array}{l}\text { Wilkinson } \\
\text { et al. [85] }\end{array}$ & Canada & Mixed & $\begin{array}{l}\text { Mean } 63 \\
\text { (range } \\
42-82)\end{array}$ & 10 & Not reported & $\begin{array}{l}\text { Pain (VAS 0-10), } \\
\text { disability (ODI 0-100) }\end{array}$ & 3 months & 100 \\
\hline $\begin{array}{l}\text { Willen et al. } \\
\text { [86] }\end{array}$ & Sweden & Mixed & $\begin{array}{l}\text { Mean } 55 \\
\quad \text { (range } \\
31-76)\end{array}$ & 21 & Not reported & $\begin{array}{l}\text { Back and leg pain (VAS } \\
0-100) \text {, disability } \\
\text { (ODI 0-100) }\end{array}$ & 31 months & 60 \\
\hline $\begin{array}{l}\text { Wong Chung- } \\
\text { Ting et al. } \\
{[87]}\end{array}$ & China & Mixed & $\begin{array}{l}\text { Mean } 60.2 \\
\text { (range } \\
38-81)\end{array}$ & 70 & $\geq 6$ months & $\begin{array}{l}\text { Pain (VAS 0-10), } \\
\text { disability (ODI 0-100) }\end{array}$ & 35 months & 60 \\
\hline Wong [88] & US & Mixed & $\begin{array}{l}\text { Mean } 73.1 \\
\text { (range } \\
63-86)\end{array}$ & 17 & Not reported & $\begin{array}{l}\text { Pain (VAS 0-10), } \\
\text { disability (ODI 0-100) }\end{array}$ & 12 months & 40 \\
\hline $\begin{array}{l}\text { Yamashita } \\
\text { et al. [89] }\end{array}$ & Japan & Mixed & $\begin{array}{l}\text { Mean } 65.9 \\
\text { (range } \\
50-81)\end{array}$ & 70 & Not reported & $\begin{array}{l}\text { Back and leg pain (VAS } \\
0-100)\end{array}$ & $\begin{array}{r}3,6,12,24, \\
36,48 \text { and } \\
60 \text { months }\end{array}$ & 60 \\
\hline $\begin{array}{l}\text { Yasar et al. } \\
\text { [90] }\end{array}$ & Turkey & Central & $\begin{array}{l}\text { Mean } 58 \\
\quad(\text { SD 11) }\end{array}$ & 125 & $\begin{array}{l}48 \% \\
\quad<24 \text { months }\end{array}$ & Disability (ODI 0-100) & $\begin{array}{l}3,12 \text { and } \\
24 \text { months }\end{array}$ & 80 \\
\hline $\begin{array}{l}\text { Yucesoy et al. } \\
\text { [91] }\end{array}$ & Turkey & Central & $\begin{array}{l}\text { Mean } 52.4 \\
\quad \text { (range } \\
15-64)\end{array}$ & 15 & 27 months & Disability (ODI 0-100) & 6 months & 60 \\
\hline $\begin{array}{l}\text { Yukawa et al. } \\
\text { [92] }\end{array}$ & US & Mixed & $\begin{array}{l}\text { Mean } 63.2 \\
\quad(\text { SD } 9.4)\end{array}$ & 62 & Not reported & $\begin{array}{l}\text { Pain (VAS 0-10), } \\
\text { disability (ODI 0-100) }\end{array}$ & 46 months & 60 \\
\hline
\end{tabular}

pain, the mean weighted score pre-surgery was 35 points (95\% CI 23.5-46.4). Three months after surgery, back pain had decreased to 16.4 (95\% CI 0.0-32.8). Small increases in back pain scores were seen at 6 months (mean 16.9; $95 \%$ CI $0.6-33.3$ ) and at 12 (mean 18; $95 \%$ CI 1.8-34.3), 24 (mean 20; $95 \%$ CI 4.3-36.3), and 60 months (mean 26.9; $95 \%$ CI 11.1-42.8).

\section{Discussion}

This systematic review included 64 cohort studies assessing post-operative outcomes in 3774 participants with lumbar spinal stenosis. The data show that most participants presented with persistent symptoms of neurological claudication, with or without back or leg pain. Prior to 


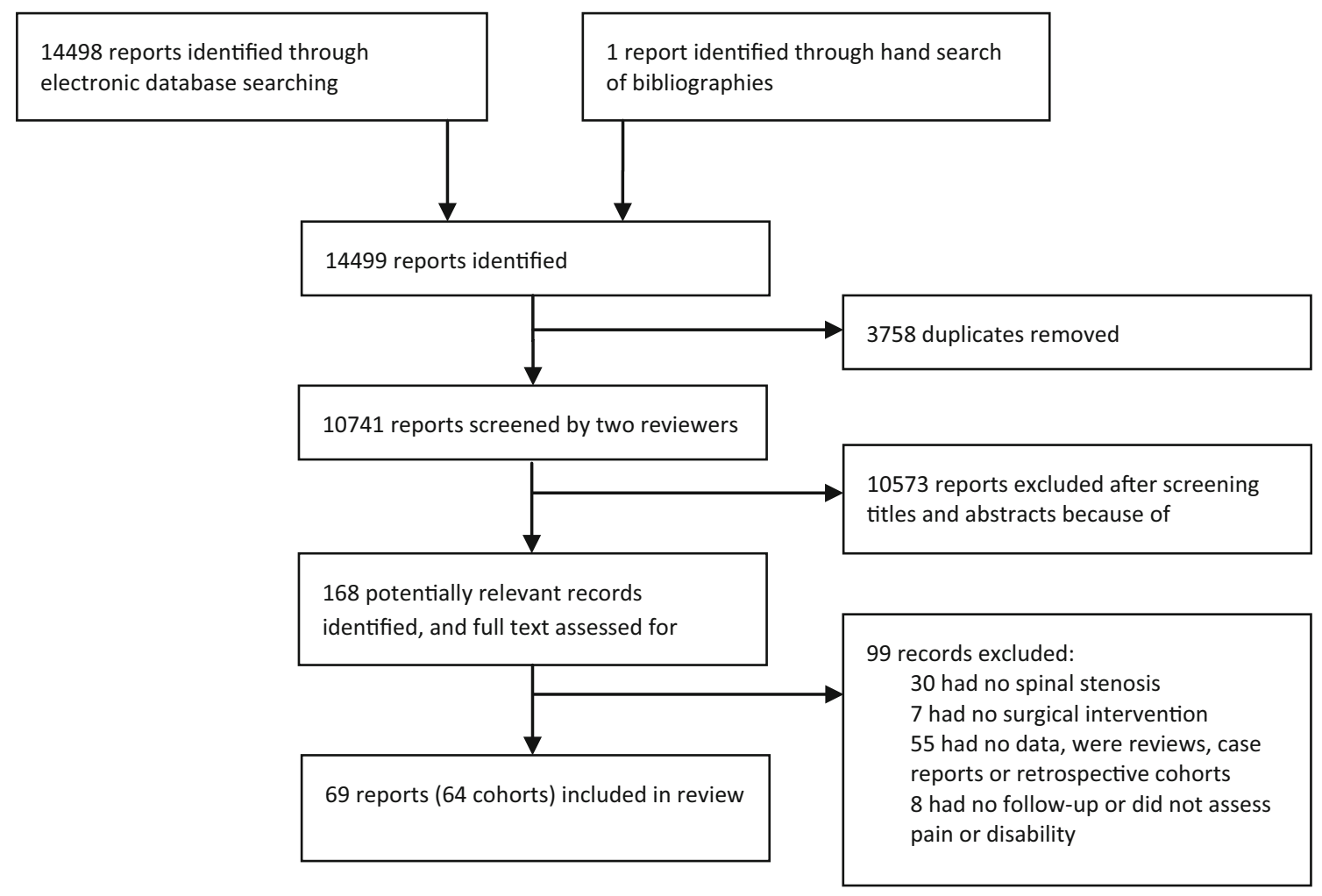

Fig. 1 Flow chart showing process of selection of studies

surgery they reported, on average, moderate levels of pain and mild disability. Typically, patients experienced substantial reductions (approximately, 50\%) in pain and disability in the first 3-month post-surgery, but little further improvement over the subsequent 5 years. On average, mild levels of pain and disability persisted at 5 years.

This is the first systematic review on the course of spinal stenosis following surgery. A quantitative approach provided precise estimates of mean pain and disability at 3,6 , 12,24 , and 60 months following surgery. The review included a large number of cohort studies of generally moderate-to-low methodological quality. The main methodological flaw was failure to recruit and clearly describe a representative sample of patients (i.e., consecutive patients presenting for care, or randomly selected patients) observed in more than half of the included studies. About a third of the studies also failed to collect or clearly describe follow-up assessments on at least $80 \%$ of the sample. One-third of the studies $(n=23 ; 33 \%)$ had to be excluded from the pooled analyses due to incomplete reporting of data. In general, sample sizes were also very small-34 studies reported data on 50 participants or less. One of the main benefits of conducting a systematic review is that it provides pooled analyses of data from many studies-data from a total of 2097 participants were included in the analysis of pain and data from 1773 participants were included in the analysis of disability. Approximately half of the studies included in the pain and disability analyses were of high methodological quality (at least $80 \%$ of total score). This gives us some confidence in the pooled estimates.

The surgical techniques varied considerably across studies. Decompression was the most prevalent type of surgery and represented $31 \%$ of the included studies. It was followed by microsurgical decompression, which represented approximately $20 \%$ of the reports. Decompression associated with fusion was performed in approximately $10 \%$ of the included studies. However, $14 \%$ of the studies reported mixed interventions and performed decompression or decompression with fusion depending on the radiological findings and/or clinical symptoms. Likewise, most studies performed a combination of single and multiple spinal-level decompression, according to the patient's diagnosis. It is possible that these variations introduced between-study heterogeneity in the pooled analysis of outcomes. However, the lack of sufficient data provided by individual studies has prevented subgroup analyses based on the types of surgical technique. However, a recent clinical trial comparing decompression surgery and decompression with fusion showed no superiority of the addition of fusion on pain, disability, walking ability, and quality-of-life up to 5 years after surgery [24]. In 

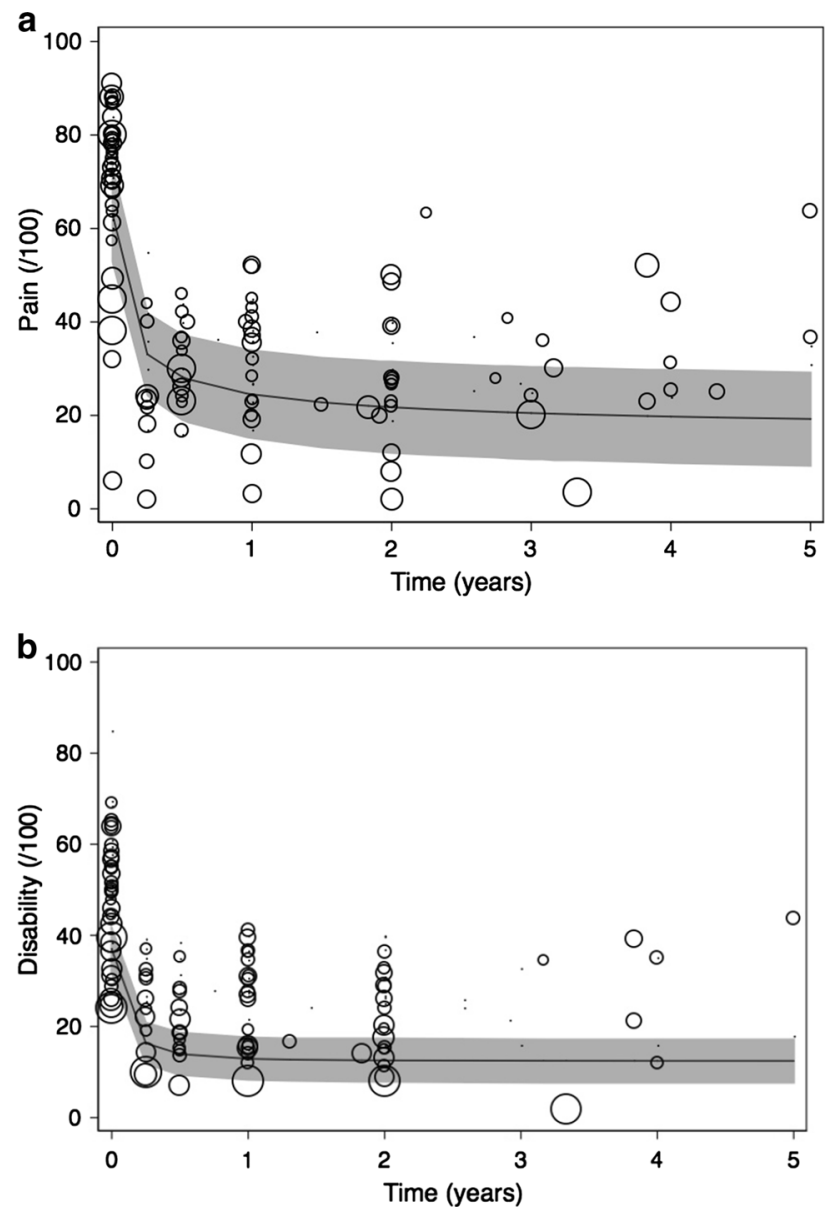

Fig. 2 Pain (a) and disability (b) outcomes after spinal stenosis surgery. Each circle represents the mean pain reported in a single study at a particular time. The area of the circle is proportional to the weight given to the study. The data have been fitted with fractional polynomial regression. The shaded area circumscribes $95 \%$ confidence interval for the regression line

addition, a systematic review compared effectiveness in regards to pain and disability among the most common surgical procedures for lumbar spinal stenosis and also reported no significant difference [8]. Therefore, it is unlikely that subgroup analyses based on the type of surgery would have yielded significantly different results.

Lumbar spinal stenosis is a highly debilitating spinal condition and its prevalence will increase over the next decades as the population ages. The number of spinal surgical procedures for spinal stenosis has also increased steadily over the years, possibly due to a scarcity of evidence on the effectiveness of non-operative management of this condition. Past research has shown that there is lack of high-quality evidence on the effectiveness of physiotherapy and non-operative interventions for patients with lumbar spinal stenosis, preventing their inclusion in clinical guideline recommendations $[25,26]$. In the US, surgery for spinal stenosis was the fastest growing type of lumbar surgical procedure between 1980 and 2000 [27, 28] and in the last decade alone, Americans have experienced a 15 -fold increase in the rate of complex fusions for lumbar spinal stenosis [6]. However, these procedures are known to be associated with important complications, such as need for cardiopulmonary resuscitation or repeat intubation [6], death [6], re-operation, and re-hospitalisation [29]. The need for re-operations following surgical procedures for lumbar spinal stenosis is not rare. In fact, the literature suggests that over one quarter of patients undergoing interspinous process implant will have a re-operation, including a revision of revision of the index procedure or the need to address the problem at a different spinal level [8]. The probability of having a second re-operation may be even greater (hazard ratio 1.58; $95 \% \mathrm{CI}, 1.41-1.76$ ). Age and presence of comorbidities, however, seem to be associated with a lower chance of having a re-operation [29]. Re-operations and hospital re-admissions are often associated with greater risk of complications and with lower satisfaction with treatment when compared to the first surgical procedure [17, 30]. Our review provides evidence that patients can expect substantial relief of pain and disability in the first 3 months after surgery, but they can also expect long-term recovery to be incomplete. This information needs to be made available to patients when discussing the indication of surgical management for spinal stenosis.

Past research also suggests that patients with lumbar spinal stenosis who also report symptoms of depression and present cardiovascular comorbidities or those resulting in impaired mobility have poorer clinical outcomes [30, 31] and greater chances of re-operation [30]. Likewise, there is compelling evidence showing that increased body weight is associated with worse self-rated quality-of-life and function in patients with lumbar spinal stenosis [32]. The role of these putative predictors could not be further evaluated in our review, as individual patient data were not available. Future studies should explore the predictive value of these as well as other patient-level characteristics on the outcomes of patients who have surgery for spinal stenosis. Most importantly, high-quality randomised trials are needed to provide robust estimates of the size of effects of surgery compared to no treatment.

\section{Conclusion}

People with spinal stenosis experience substantial reductions in pain and disability in the first 3 months after surgery. Little further improvement is observed in the following 5 years. 
Acknowledgments MLF is a Sydney Medical Foundation Fellow/ Sydney Medical School, CGM is supported by the Australian Research Council, and RDH is supported by the National Health and Medical Research Council of Australia.

\section{Compliance with ethical standards}

Conflict of interest All authors have no conflict of interest. Authors have full control of all primary data and agree to allow the journal to review their data if requested.

Funding None.

\section{References}

1. Butt S, Saifuddin A (2005) The imaging of lumbar spondylolisthesis. Clin Radiol 60(5):533-546. doi:10.1016/j.crad.2004.07. 013

2. Comer CM, Redmond AC, Bird HA, Conaghan PG (2009) Assessment and management of neurogenic claudication associated with lumbar spinal stenosis in a UK primary care musculoskeletal service: a survey of current practice among physiotherapists. BMC Musculoskel Disord 10:121. doi:10.1186/ 1471-2474-10-121

3. Winter CC, Brandes M, Muller C, Schubert T, Ringling M, Hillmann A, Rosenbaum D, Schulte TL (2010) Walking ability during daily life in patients with osteoarthritis of the knee or the hip and lumbar spinal stenosis: a cross sectional study. BMC Musculoskelet Disord 11:233. doi:10.1186/1471-2474-11-233

4. Otani K, Kikuchi S, Yabuki S, Igarashi T, Nikaido T, Watanabe K, Konno S (2013) Lumbar spinal stenosis has a negative impact on quality of life compared with other comorbidities: an epidemiological cross-sectional study of 1862 community-dwelling individuals. Sci World J 2013:590652. doi:10.1155/2013/590652

5. Ishimoto Y, Yoshimura N, Muraki S, Yamada H, Nagata K, Hashizume H, Takiguchi N, Minamide A, Oka H, Kawaguchi H, Nakamura K, Akune T, Yoshida M (2013) Associations between radiographic lumbar spinal stenosis and clinical symptoms in the general population: the Wakayama Spine Study. Osteoarthr Cartil OARS 21(6):783-788. doi:10.1016/j.joca.2013.02.656

6. Deyo RA, Mirza SK, Martin BI, Kreuter W, Goodman DC, Jarvik JG (2010) Trends, major medical complications, and charges associated with surgery for lumbar spinal stenosis in older adults. JAMA 303(13):1259-1265. doi:10.1001/jama.2010.338

7. Harris IA, Dao AT (2009) Trends of spinal fusion surgery in Australia: 1997-2006. ANZ J Surg 79(11):783-788. doi:10.1111/ j.1445-2197.2009.05095.x

8. Machado G, Ferreira P, Harris I, Pinheiro M, Koes B, van Tulder M, Rzewuska M, Maher C, Ferreira M (2015) Effectiveness of surgery for lumbar spinal stenosis: a systematic review and metaanalysis. PLoS One 10(3):e0122800

9. Amundsen T, Weber H, Lilleas F, Nordal HJ, Abdelnoor M, Magnaes B (1995) Lumbar spinal stenosis. Clinical and radiologic features. Spine (Phila Pa 1976) 20(10):1178-1186

10. Lohman CM, Tallroth K, Kettunen JA, Lindgren KA (2006) Comparison of radiologic signs and clinical symptoms of spinal stenosis. Spine (Phila Pa 1976) 31(16):1834-1840. doi:10.1097/ 01.brs.0000227370.65573.ac

11. Gunzburg R, Keller TS, Szpalski M, Vandeputte K, Spratt KF (2003) A prospective study on CT scan outcomes after conservative decompression surgery for lumbar spinal stenosis. J Spinal Disord Tech 16(3):261-267
12. Altman DG (2001) Systematic reviews of evaluations of prognostic variables. BMJ 323(7306):224-228

13. Menezes CL, Maher CG, Hancock MJ, McAuley JH, Herbert RD, Costa LO (2012) The prognosis of acute and persistent low-back pain: a meta-analysis. CMAJ 184(11):E613-E624. doi:10.1503/ cmaj. 111271

14. Royston P, Sauerbrei W (2008) Multivariable model-building: a pragmatic approach to regression analysis based on fractional polynomials for modelling continuous variables. Wiley, Chichester

15. Atlas S, Deyo R, Keller R, Chapin A, Patrick D, Long J, Singer D (1996) The maine lumbar spine study, Part II. 1-year outcomes of surgical and nonsurgical management of sciatica. Spine 21(15): $1777-1786$

16. Atlas SJ, Keller RB, Robson D, Deyo RA, Singer DE (2000) Surgical and nonsurgical management of lumbar spinal stenosis: four-year outcomes from the maine lumbar spine study. Spine 25(5):556-562

17. Atlas SJ, Keller RB, Wu YA, Deyo RA, Singer DE (2005) Longterm outcomes of surgical and nonsurgical management of lumbar spinal stenosis: 8 to 10 year results from the maine lumbar spine study. Spine 30(8):936-943

18. Richter A, Schutz C, Hauck M, Halm H (2010) Does an interspinous device (Coflex) improve the outcome of decompressive surgery in lumbar spinal stenosis? One-year follow up of a prospective case control study of 60 patients. Eur Spine J 19(2):283-289

19. Richter A, Halm HF, Hauck M, Quante M (2014) Two-year follow-up after decompressive surgery with and without implantation of an interspinous device for lumbar spinal stenosis: a prospective controlled study. J Spinal Disord Tech 27(6):336-341

20. Schaeren S, Broger I, Jeanneret B (2008) Minimum four-year follow-up of spinal stenosis with degenerative spondylolisthesis treated with decompression and dynamic stabilization. Spine 33(18):E636-E642

21. Schnake KJ, Schaeren S, Jeanneret B (2006) Dynamic stabilization in addition to decompression for lumbar spinal stenosis with degenerative spondylolisthesis. Spine 31(4):442-449

22. Bednar DA (2002) Surgical management of lumbar degenerative spinal stenosis with spondylolisthesis via posterior reduction with minimal laminectomy. J Spinal Disord 15(2):105-109

23. Ha KY, Seo JY, Kwon SE, Son IN, Kim KW, Kim YH (2013) Posterior dynamic stabilization in the treatment of degenerative lumbar stenosis: validity of its rationale. J Neurosurg Spine 18(1):24-31. doi:10.3171/2012.9.SPINE12392

24. Forsth P, Olafsson G, Carlsson T, Frost A, Borgstrom F, Fritzell P, Ohagen P, Michaelsson K, Sanden B (2016) A randomized, controlled trial of fusion surgery for lumbar spinal stenosis. N Engl J Med 374(15):1413-1423. doi:10.1056/NEJMoa1513721

25. Ammendolia C, Stuber KJ, Rok E, Rampersaud R, Kennedy CA, Pennick V, Steenstra IA, de Bruin LK, Furlan AD (2013) Nonoperative treatment for lumbar spinal stenosis with neurogenic claudication. Cochrane Database Syst Rev 8:CD010712. doi:10. 1002/14651858.CD010712

26. Macedo LG, Hum A, Kuleba L, Mo J, Truong L, Yeung M, Battie MC (2013) Physical therapy interventions for degenerative lumbar spinal stenosis: a systematic review. Phys Ther 93(12):1646-1660. doi:10.2522/ptj.20120379

27. Taylor VM, Deyo RA, Cherkin DC, Kreuter W (1994) Low back pain hospitalization. Recent US trends and regional variations. Spine (Phila Pa 1976) 19(11):1207-1212

28. Deyo RA, Gray DT, Kreuter W, Mirza S, Martin BI (2005) US trends in lumbar fusion surgery for degenerative conditions. Spine (Phila Pa 1976) 30(12):1441-1445 
29. Deyo RA, Martin BI, Kreuter W, Jarvik JG, Angier H, Mirza SK (2011) Revision surgery following operations for lumbar stenosis. J Bone Jt Surg 93(21):1979-1986. doi:10.2106/JBJS.J.01292

30. Aalto TJ, Malmivaara A, Kovacs F, Herno A, Alen M, Salmi L, Kroger H, Andrade J, Jimenez R, Tapaninaho A, Turunen V, Savolainen S, Airaksinen O (2006) Preoperative predictors for postoperative clinical outcome in lumbar spinal stenosis: systematic review. Spine 31(18):E648-E663. doi:10.1097/01.brs. 0000231727.88477.da

31. McKillop AB, Carroll LJ, Battie MC (2014) Depression as a prognostic factor of lumbar spinal stenosis: a systematic review. Spine J 14(5):837-846. doi:10.1016/j.spinee.2013.09.052

32. Knutsson B, Michaelsson K, Sanden B (2013) Obesity is associated with inferior results after surgery for lumbar spinal stenosis: a study of 2633 patients from the Swedish spine register. Spine 38(5):435-441

33. Aleem IS, Rampersaud YR (2014) Elderly patients have similar outcomes compared to younger patients after minimally invasive surgery for spinal stenosis. Clin Orthop 472:1824-1830

34. Anjarwalla NK, Brown LC, McGregor AH (2007) The outcome of spinal decompression surgery 5 years on. Eur Spine $\mathrm{J}$ $16: 1842-1847$

35. Athiviraham A, Yen D (2007) Is spinal stenosis better treated surgically or nonsurgically? Clin Orthop 458:90-93

36. Beyer F, Yagdiran A, Neu P, Kaulhausen T, Eysel P, Sobottke R (2013) Percutaneous interspinous spacer versus open decompression: a 2-year follow-up of clinical outcome and quality of life. Eur Spine J 22:2015-2021

37. Bhadra AK, Raman AS, Tucker S, Noordeen HH (2008) Interspinous implant in lumbar spinal stenosis: a prospective cohort. Eur J Orthop Surg Tr 18:489-493

38. Castro-Menendez M, Bravo-Ricoy JA, Casal-Moro R, Hernandez-Blanco M, Jorge-Barreiro FJ (2009) Midterm outcome after microendoscopic decompressive laminotomy for lumbar spinal stenosis: 4-year prospective study. Neurosurgery 65:100-110

39. Cavagna R, Tournier C, Aunoble S, Bouler JM, Antonietti P, Ronai $\mathrm{M}$ et al (2008) Lumbar decompression and fusion in elderly osteoporotic patients: a prospective study using less rigid titanium rod fixation. J Spinal Disord Tech 21:86-91

40. Cavusoglu H, Turkmenogulu O, Kaya RA, Tuncer C, Colak I, Sahin Y et al (2007) Efficacy of unilateral laminectomy for bilateral decompression in lumbar spinal stenosis. Turk Neurosurg 17:100-108

41. Chopko BW (2013) Long-term results of percutaneous lumbar decompression for LSS: two-year outcomes. Clin J Pain 29(11):939-943

42. Colak A, Topuz K, Kutlay M, Kaya S, Simsek H, Cetinkal A et al (2008) A less invasive surgical approach in the lumbar lateral recess stenosis: direct approach to the medial wall of the pedicle. Eur Spine J 17:1745-1751

43. Datta G, Gnanalingham KK, Peterson D, Mendoza N, O’Neill K, Van Dellen J et al (2004) Back pain and disability after lumbar laminectomy: is there a relationship to muscle retraction? Neurosurgery 54:1413-1420

44. Deer TR, Kim CK, Bowman RG, Ranson MT, Yee BS (2012) Study of percutaneous lumbar decompression and treatment algorithm for patients suffering from neurogenic claudication. Pain Physician 15:451-460

45. Delank KS, Eysel P, Zollner J, Drees P, Nafe B, Rompe JD (2002) Undercutting decompression versus laminectomy. Clinical and radiological results of a prospective controlled trial. Orthopade 31:1048-1057

46. El-Abed K, Barakat M, Ainscow D (2011) Multilevel lumbar spinal stenosis decompression: midterm outcome using a modified hinge osteotomy technique. J Spinal Disord Tech 24:376-380
47. Endres S (2011) Instrumented posterolateral fusion - clinical and functional outcome in elderly patients. Ger Med Sci 9:Doc09

48. Frazier DD, Lipson SJ, Fossel AH, Katz JN (1997) Associations between spinal deformity and outcomes after decompression for spinal stenosis. Spine 22:2025-2029

49. Fu YS, Zeng BF, Xu JG (2008) Long-term outcomes of two different decompressive techniques for lumbar spinal stenosis. Spine 33:514-518

50. Greiner-Perth R, Boehm H, Allam Y, El-Saghir H (2004) A less invasive approach technique for operative treatment of lumbar canal stenosis. Technique and preliminary results. Zentralbl Neurochir 65:185-190

51. Haro H, Maekawa S, Hamada Y (2008) Prospective analysis of clinical evaluation and self-assessment by patients after decompression surgery for degenerative lumbar canal stenosis. Spine $\mathbf{J}$ $8: 380-384$

52. Herno A, Airaksinen O, Saari T (1993) Long-term results of surgical treatment of lumbar spinal stenosis. Spine 18:1471-1474

53. Ikuta K, Tono O, Oga M (2008) Clinical outcome of microendoscopic posterior decompression for spinal stenosis associated with degenerative spondylolisthesis-minimum 2-year outcome of 37 patients. Minim Invasive Neurosurg 51:267-271

54. Jakola AS, Sorlie A, Gulati S, Nygaard OP, Lydersen S, Solberg $\mathrm{T}$ (2010) Clinical outcomes and safety assessment in elderly patients undergoing decompressive laminectomy for lumbar spinal stenosis: a prospective study. BMC Surg 10:34

55. Kaner T, Sasani M, Oktenoglu T, Cosar M, Ozer AF (2010) Clinical outcomes after posterior dynamic transpedicular stabilization with limited lumbar discectomy: Carragee classification system for lumbar disc herniations. SAS Journal 4:92-97

56. Kim HJ, Lee HM, Chun HJ, Kang KT, Kim HS, Park JO et al (2009) Restoration of bone turnover rate after decompression surgery in patients with symptomatic lumbar spinal stenosis: preliminary report. Spine 34:E635-E639

57. Kim HY, Choi BW (2013) Change of radiological parameters after interspinous implantation (X-stop $\left.{ }^{\circledR}\right)$ in degenerative spinal stenosis. Eur J Orthop Surg Trauma 23:281-285

58. Komp M, Hahn P, Merk H, Godolias G, Ruetten S (2011) Bilateral operation of lumbar degenerative central spinal stenosis in full-endoscopic interlaminar technique with unilateral approach: prospective 2-year results of 74 patients. J Spinal Disord Tech 24:281-287

59. Kong DS, Kim ES, Eoh W (2007) One-year outcome evaluation after interspinous implantation for degenerative spinal stenosis with segmental instability. J Korean Med Sci 22:330-335

60. Kuchta J, Sobottke R, Eysel P, Simons P (2009) Two-year results of interspinous spacer (X-Stop) implantation in 175 patients with neurologic intermittent claudication due to lumbar spinal stenosis. Eur Spine J 18:823-829

61. Mannion RJ, Guilfoyle MR, Efendy J, Nowitzke AM, Laing RJ, Wood MJ (2012) Minimally invasive lumbar decompression: long-term outcome, morbidity, and the learning curve from the first 50 cases. J Spinal Disord Tech 25:47-51

62. Mekhail N, Costandi S, Abraham B, Samuel SW (2012) Functional and patient-reported outcomes in symptomatic lumbar spinal stenosis following percutaneous decompression. Pain Pract $12: 417-425$

63. Mekhail N, Vallejo R, Coleman MH, Benyamin RM (2012) Long-term results of percutaneous lumbar decompression mild for spinal stenosis. Pain Pract 12:184-193

64. Ng LC, Tafazal S, Sell P (2007) The effect of duration of symptoms on standard outcome measures in the surgical treatment of spinal stenosis. Eur Spine J 16:199-206

65. Ohtori S, Yamashita M, Yamauchi K, Inoue G, Koshi T, Suzuki $M$ et al (2010) Change in Modic type 1 and 2 signals after posterolateral fusion surgery. Spine 35:1231-1235 
66. Paker N, Turkmen C, Bugdayci D, Tekdos D, Erbil M (2005) Comparison of conservative and surgical treatment results in lumbar spinal stenosis. Turk Neurosurg 15:182-184

67. Palmer S, Davison L (2012) Minimally invasive surgical treatment of lumbar spinal stenosis: two-year follow-up in 54 patients. Surg Neurol Int 3:41

68. Panagiotis ZE, Athanasios K, Panagiotis D, Minos T, Charis M, Elias L (2006) Functional outcome of surgical treatment for multilevel lumbar spinal stenosis. Acta Orthop 77:670-676

69. Pao JL, Chen WC, Chen PQ (2009) Clinical outcomes of microendoscopic decompressive laminotomy for degenerative lumbar spinal stenosis. Eur Spine J 18:672-678

70. Papavero L, Thiel M, Fritzsche E, Kunze C, Westphal M, Kothe R (2009) Lumbar spinal stenosis: prognostic factors for bilateral microsurgical decompression using a unilateral approach. Neurosurgery $65: 182-187$

71. Parker SL, Fulchiero EC, Davis BJ, Adogwa O, Aaronson OS, Cheng JS et al (2011) Cost-effectiveness of multilevel hemilaminectomy for lumbar stenosis-associated radiculopathy. Spine J 11:705-711

72. Parlato C, Iavarone A, Gentile M, Albanese R, Moraci A (2013) Outcome of lumbar intervertebral foraminal stenosis surgery and depression. Eur Neurol 69:304-308

73. Postacchini R, Ferrari E, Cinotti G, Menchetti PPM, Postacchini F (2011) Aperius interspinous implant versus open surgical decompression in lumbar spinal stenosis. Spine J 11:933-939

74. Reyes-Sanchez A, Sanchez-Bringas G, Zarate-Kalfopulos B, Alpizar-Aguirre A, Lara-Padilla E, Rosales-Olivares LM (2013) [Transpedicular dynamics stabilization in the treatment of lumbar stenosis. Fourth years follow-up]. Cir Cir 81:48-54

75. Schulte TL, Schubert T, Winter C, Brandes M, Hackenberg L, Wassmann $\mathrm{H}$ et al (2010) Step activity monitoring in lumbar stenosis patients undergoing decompressive surgery. Eur Spine J 19:1855-1864

76. Shabat S, Arinzon Z, Folman Y, Leitner J, David R, Pevzner E et al (2008) Long-term outcome of decompressive surgery for lumbar spinal stenosis in octogenarians. Eur Spine J 17:193-198

77. Shabat S, Miller LE, Block JE, Gepstein R (2011) Minimally invasive treatment of lumbar spinal stenosis with a novel interspinous spacer. Clin Interv Aging 6:227-233

78. Sigmundsson FG, Kang XP, Jonsson B, Stromqvist B (2012) Prognostic factors in lumbar spinal stenosis surgery. Acta Orthop 83:536-542

79. Sinikallio S, Lehto SM, Aalto $\mathrm{T}$, Airaksinen $\mathrm{O}$, Kroger $\mathrm{H}$, Viinamaki H (2010) Depressive symptoms during rehabilitation period predict poor outcome of lumbar spinal stenosis surgery: a two-year perspective. BMC Musculoskel Dis 11:152

80. Sobottke R, Aghayev E, Roder C, Eysel P, Delank SK, Zweig T (2012) Predictors of surgical, general and follow-up complications in lumbar spinal stenosis relative to patient age as emerged from the Spine Tango Registry. Eur Spine J 21:411-417

81. Stromqvist BH, Berg S, Gerdhem P, Johnsson R, Moller A, Sahlstrand T et al (2013) X-stop versus decompressive surgery for lumbar neurogenic intermittent claudication: randomized controlled trial with 2-year follow-up. Spine 38:1436-1442

82. Surace MF, Fagetti A, Fozzato S, Cherubino P (2012) Lumbar spinal stenosis treatment with Aperius perclid interspinous system. Eur Spine J 21(1):S69-S74

83. Tenhula J, Lenke LG, Bridwell KH, Gupta P, Riew D (2000) Prospective functional evaluation of the surgical treatment of neurogenic claudication in patients with lumbar spinal stenosis. J Spinal Disord 13:276-282

84. Westergaard L, Hauerberg J, Springborg JB (2009) Outcome after surgical treatment for lumbar spinal stenosis: the lumbar extension test is not a predictive factor. Spine 34:E930-E935

85. Wilkinson JS, Fourney DR (2012) Failure of percutaneous remodeling of the ligamentum flavum and lamina for neurogenic claudication. Neurosurgery 71:86-91

86. Willen J, Wessberg PJ, Danielsson B (2008) Surgical results in hidden lumbar spinal stenosis detected by axial loaded computed tomography and magnetic resonance imaging: an outcome study. Spine 33:E109-E115

87. Wong Chung-Ting M, Chan Pak-Ho A, Cheung KK (2012) A prospective study on the outcome of degenerative lumbar spinal stenosis treated with open laminotomy. J Orthop Trauma Rehab 16:62-65

88. Wong WHM (2012) Mild interlaminar decompression for the treatment of lumbar spinal stenosis: procedure description and case series with 1-year follow-up. Clin J Pain 28:534-538

89. Yamashita K, Ohzono K, Hiroshima K (2006) Five-year outcomes of surgical treatment for degenerative lumbar spinal stenosis: a prospective observational study of symptom severity at standard intervals after surgery. Spine 31:1484-1490

90. Yasar B, Simsek S, Er U, Yigitkanli K, Eksioglu E, Altug T et al (2009) Functional and clinical evaluation for the surgical treatment of degenerative stenosis of the lumbar spinal canal: clinical article. J Neurosurg Spine 11:347-352

91. Yucesoy K, Ozer E (2002) Inverse laminoplasty for the treatment of lumbar spinal stenosis. Spine 27:E316-E320

92. Yukawa Y, Lenke LG, Tenhula J, Bridwell KH, Riew KD, Blanke K (2002) A comprehensive study of patients with surgically treated lumbar spinal stenosis with neurogenic claudication. J Bone Joint Surg 84-A:1954-1959

93. Mlyavykh S, Ludwig SC, Mobasser JP, Kepler CK, Anderson G (2013) Twelve-month results of a clinical pilot study utilizing pedicle-lengthening osteotomy for the treatment of lumbar spinal stenosis. J Neurosurg Spine 18(4):347-355. doi:10.3171/2012.11. SPINE12402 